\title{
Trastorno por atracón asociado a depresión y ansiedad en pacientes con diabetes tipo 2
}

\author{
Binging eating disorder associated with depression \\ and anxiety in patients with diabetes type 2
}

Fátima Ireri Ramos Salazar Díaz*

Citar como: Ramos SDFI. Trastorno por atracón asociado a depresión y ansiedad en pacientes con diabetes 2. Acta Med. 2020; 18 (4): 367-372. https://dx.doi.org/10.35366/97262

\section{Resumen}

La diabetes mellitus tiene una alta incidencia e incremento en población mexicana. Al igual que otras enfermedades tiene componentes en la psique, lo que conlleva comorbilidades psiquiátricas como depresión, ansiedad y trastorno de atracones. Este último es importante debido a que la dieta es un factor indispensable para el control de la glucemia en pacientes con diabetes mellitus. Material y métodos: Estudio observacional, descriptivo y transversal. Se estudiaron pacientes con diabetes mellitus tipo 2 (DM2) bajo tratamiento en la Clínica Especializada en el Manejo Integral de la Diabetes Iztapalapa, a quienes se les aplicó The Binge Eating Scale (16-SF), cuestionario de depresión de Hamilton y el inventario de ansiedad de Beck. Resultados: Se incluyeron 98 pacientes con DM2, con una edad promedio de $55.92 \pm 11.28$ años. Se encontraron prevalencias de depresión de $71.4 \%$, ansiedad $42.9 \%$ y trastorno de atracones de $28.66 \%$. El trastorno de atracones se relacionó significativamente con la presencia de depresión y ansiedad $(p<0.0001)$. Conclusión: La diabetes mellitus se caracteriza también por presentar alteraciones de tipo psiquiátrico como depresión, ansiedad y trastorno de atracones; este último es perpetuado por el desarrollo de depresión y ansiedad que se han descrito como posibles complicaciones biológicas de la diabetes mellitus.

Palabras clave: Diabetes mellitus, depresión, ansiedad, atracón.

\section{Abstract}

Diabetes mellitus is one of the diseases with the highest incidence in the Mexican population and whose incidence is growing. However, as any disease that affects the human being also has components at the level of the psyche; which can be related to the development of psychiatric comorbidities such as depression, anxiety and binge eating disorder. The latest is important because diet is an indispensable factor for glycemic control in patients with diabetes mellitus. Material and methods: Observational, extensive and transversal study. Patients with type 2 diabetes mellitus were studied under medical treatment, to whom The Binge Eating Scale instrument (16-SF), Hamilton depression questionnaire and Beck's anxiety inventory were applied. Results: 98 cases of patients with diabetes mellitus with an average age of 55.92 \pm 11.28 years were included. A prevalence of depression of $71.4 \%$, anxiety $42.9 \%$ and binge eating disorder of $28.66 \%$ was found. Binge eating disorder was significantly related to the presence of depression and anxiety $(p<0.0001)$. Conclusion: Diabetes mellitus is also characterized by presenting psychiatric disorders such as depression, anxiety and binge eating disorder; that is perpetuated by the development of depression and anxiety that have been described as possible biological complications of diabetes mellitus.

Keywords: Diabetes mellitus, depression, anxiety, binge eating.

\section{www medigraphic.org.mx}

* Médico residente de Psiquiatría, Instituto Nacional de Psiquiatría “Dr. Ramón de la Fuente Muñiz", Facultad Mexicana de Medicina de la Universidad La Salle. Ciudad de México, México.
Correspondencia:

Dra. Fátima Ireri Ramos Salazar Díaz Correo electrónico: 80bm00@gmail.com

Aceptado: 13-02-2020.

www.medigraphic.com/actamedica

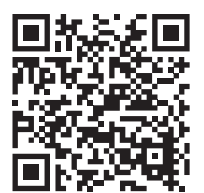




\section{INTRODUCCIÓN}

La diabetes mellitus es una enfermedad caracterizada por un incremento en los niveles de glucosa en sangre y la incapacidad del organismo para regularlos. ${ }^{1}$ Ésta se ha convertido en la epidemia del siglo XX, ya que sólo en México contribuye con $12 \%$ de las muertes anuales. ${ }^{2}$

Dicha enfermedad no sólo se limita a los cambios en la glucemia, sino que también se ha caracterizado por el desarrollo de múltiples comorbilidades que se asocian con la pérdida de la función, disminución en la calidad y esperanza de vida como la falla renal, neuropatía y retinopatía diabética, por mencionar algunas. ${ }^{3}$

Sin embargo, dentro de sus comorbilidades se puede destacar la presencia de aquéllas que afectan la salud mental o son de orden psiquiátrico, siendo los trastornos depresivos los más estudiados. ${ }^{4}$ Sin dejar a un lado que se ha observado una asociación en pacientes con diabetes mellitus con los trastornos de alimentación como la obesidad que es la más habitual. ${ }^{5,6}$

Prueba de ello es que se ha asociado la alimentación compulsiva con la obesidad y el desarrollo de diabetes tipo $2,{ }^{7}$ lo que no descartaría un trastorno de alimentación tipo atracón en los pacientes con diabetes tipo 2, el cual se entiende acorde al DSM 5 como la ingesta superior en cantidad a lo que la mayoría de las personas ingeriría en un periodo similar y en circunstancias parecidas así como la sensación de pérdida de control sobre la ingesta de alimentos durante dicho periodo. ${ }^{8,9}$ Para la evaluación del trastorno de atracones se han desarrollado diversas escalas como The Binge Eating Scale (16-SF) (BES); sin embargo, poco se ha estudiado sobre el atracón y su asociación directa con la diabetes tipo $2 .{ }^{10,11}$

\section{MATERIAL Y MÉTODOS}

Estudio prospectivo, transversal, descriptivo, observacional y analítico, en el cual se incluyeron pacientes con diagnóstico de diabetes mellitus tipo 2 que se encontraban bajo tratamiento en la Clínica Especializada en el Manejo Integral de la Diabetes Iztapalapa al momento de la evaluación. A dichos pacientes se les invitó a participar de manera voluntaria en un área de consulta externa entre julio y octubre de 2019. Una vez que aceptaron participar, se les aplicaron los siguientes cuestionarios:

- The Binge Eating Scale (16-SF) (BES): cuestionario desarrollado en 1982 que se integra de 16 preguntas para la evaluación del trastorno de atracones considerando el aspecto emocional, el sentido de culpa o vergüenza. Las preguntas son de tipo escala de Likert con valores de 0 a 3, con un puntaje de 18-26 se considera la presencia de un trastorno moderado y con más de 27 puntos un trastorno grave. ${ }^{12,13}$

- Cuestionario de depresión de Hamilton: la escala de Hamilton para depresión se integra de 17 preguntas con respuestas de escala tipo Likert con un puntaje que puede ser de 0 a 2 y de 0 a 4 . Resultados con un puntaje menor de siete significa no deprimido, depresión leve de ocho a doce, depresión moderada de 13-17, depresión grave de 18-22 y muy grave $\geq 23$ puntos. $^{14}$

- Inventario de ansiedad de Beck: es un instrumento de 21 preguntas que evalúan la gravedad de las categorías sintomáticas y conductuales de la ansiedad. ${ }^{15}$ Cada pregunta tiene respuestas tipo Likert de 0-3; si el puntaje sumado es menor de 21 se considera ansiedad leve, de 22-35 ansiedad moderada y mayor de 36 ansiedad grave. ${ }^{16}$

Consideraciones éticas. Todos los pacientes firmaron un consentimiento informado sobre su libre participación, confidencialidad de datos y posibilidad de abandono del estudio de manera voluntaria.

El análisis estadístico se llevó a cabo mediante el uso del programa IBM SPSS v 25 (USA). Para las variables cualitativas se empleó cálculo de frecuencias y porcentajes, mientras que para las cuantitativas se emplearon medidas de tendencia central. La normalidad de la muestra fue evaluada con la prueba de Kolmogorov-Smirnov y la asociación entre dos variables cualitativas se analizó mediante la prueba de $\chi^{2}$ de Pearson; por último, para comparar dos variables cuantitativas se empleó la prueba de t-Student (paramétrica). Se consideró significativo el resultado cuando el valor de $\mathrm{p} \leq 0.05$.

\section{RESULTADOS}

El estudio se compuso de 98 casos con una edad promedio de $55.92 \pm 11.28$ años, de los cuales $51 \%$ fueron mujeres y $49 \%$ hombres; dicha población presenta una distribución normal con base en la prueba de Kolmogorov-Smirnov. No se encontró diferencia significativa entre la edad de hombres y mujeres $(p=0.7716)$. El tiempo de evolución de la enfermedad en promedio fue de $17.42 \pm 13.38$ años.

Respecto al tratamiento que seguía esta población, $78.6 \%$ se encontraba con un tratamiento mixto consistente en hipoglucemiantes orales e insulina, $7.1 \%$ con tratamiento de insulina exclusiva y $14.3 \%$ sólo con hipoglucemiantes orales.

En cuanto a las condiciones de salud mental evaluadas, se encontró que $28.6 \%$ de la población presentó trastorno de atracones, $71.4 \%$ mostró trastorno depresivo y $42.9 \%$ trastorno de ansiedad.

Por otro lado, se realizó la evaluación sobre la gravedad de trastorno de atracones según los resultados del instru- 
mento BES, observando que $50 \%$ de los casos presentó un trastorno de atracones leve y $50 \%$ grave.

Referente al trastorno depresivo de los casos hallados, $42.85 \%$ se encontraba en depresión leve, $21.42 \%$ depresión moderada y $7.14 \%$ fue identificado con depresión grave. La edad promedio de la población con depresión fue de $58.3 \pm 7.41$ años y el grupo sin depresión tuvo una media de edad de $50 \pm 15.41$ años con una diferencia significativa entre ambos grupos $(p=0.0005)$. Con relación a la gravedad del trastorno de ansiedad, $100 \%$ de los casos la presentaba en un grado leve.

Posteriormente, se realizó un análisis entre la presencia de trastorno de atracones y la edad, sexo, tratamiento, tiempo de evolución, presencia de depresión y ansiedad así como la existencia de diferencia entre la edad y los años de evolución de la población con y sin trastorno de atracones (Tabla 1).

Se realizó un análisis de correlación entre la variable en análisis y la presencia de trastorno de atracones o no, a su vez se llevó a cabo un análisis comparativo de las variables cuantitativas del grupo de pacientes con trastorno de atracones y sin él.

El resultado de los puntajes de cada escala entre el grupo con trastorno de atracones o sin él fueron evaluados y comparados mediante prueba de t-Student como se muestra en la Tabla 2.

Así como se encontró una relación significativa entre la presencia de depresión y ansiedad con el trastorno de atracones, el nivel de depresión y el nivel de ansiedad se relacionan significativamente con la presencia de atracones y nivel de trastorno de atracones ( $p \leq 0.0001$ para ambos casos).

\section{DISCUSIÓN}

Acorde con la distribución de la población por sexo detectada en nuestro estudio, se observó que es similar al comportamiento de la población mexicana según lo señalado en los reportes del INEGI (Instituto Nacional de Estadística, Geografía e Informática). ${ }^{17}$ Sin embargo, dicho hallazgo no sólo está limitado al comportamiento poblacional, sino que existe mayor prevalencia de diabetes en mujeres que en hombres, lo que implica que en la población estudiada era de esperarse mayor presencia de mujeres que de hombres. También la edad de la población diabética observada en nuestro estudio concuerda con los datos epidemiológicos publicados para México. ${ }^{2,18}$ A su vez el tiempo de evolución de la diabetes en la población mexicana es de más de cinco años en al menos $50 \%$ de la población con la enfermedad, por lo que nuestros hallazgos en relación al tiempo de evolución de la enfermedad es concordante con dicho reporte. ${ }^{2}$ Motivo por el cual podemos inferir que la población estudiada es una muestra que reúne las características de la población diabética mexicana.

En cuanto al tratamiento, se han descrito diversos esquemas con o sin insulina; sin embargo, esta última en

Tabla 1: Comparación entre la población con y sin presencia de trastorno de atracones.

\begin{tabular}{|c|c|c|c|c|c|}
\hline & $\begin{array}{c}\text { Con } \\
\text { atracón }\end{array}$ & $\begin{array}{c}\text { Sin } \\
\text { atracón }\end{array}$ & $p$ & OR & IC 95\% \\
\hline Sexo & & & $0.002^{*}$ & 0.222 & $0.083-0.592$ \\
\hline Hombres & $14.29 \%$ & $85.71 \%$ & & 0.417 & $0.213-0.814$ \\
\hline Mujeres & $42.86 \%$ & $57.14 \%$ & & 1.875 & $1.311-2.682$ \\
\hline Edad (años) & $60.75 \pm 3.94$ & $54 \pm 12.81$ & $0.3314^{\star *}$ & & \\
\hline Años de evolución & $20.25 \pm 7.71$ & $16.3 \pm 15.3$ & $0.637^{\star \star}$ & & \\
\hline Tratamiento & & & $0.022^{*}$ & & \\
\hline Hipoglucemiantes & $49 \%$ & $48 \%$ & & & \\
\hline Insulina & $0 \%$ & $100 \%$ & & & \\
\hline Mixto & $27.27 \%$ & $72.73 \%$ & & & \\
\hline Depresión & & & $<0.0001^{*}$ & 1.667 & $1.337-2.018$ \\
\hline Sí & $40 \%$ & $60 \%$ & & & \\
\hline No & $0 \%$ & $100 \%$ & & & \\
\hline Ansiedad & & & $<0.0001^{*}$ & 5.0 & 3.13-7.98 \\
\hline Sí & $100 \%$ & $0 \%$ & & & \\
\hline No & $20 \%$ & $80 \%$ & & & \\
\hline
\end{tabular}




\begin{tabular}{llcc|}
\hline \multicolumn{3}{c}{$\begin{array}{c}\text { Tabla 2: Comparación del puntaje } \\
\text { obtenido en cada prueba aplicada. }\end{array}$} \\
\hline & Con trastorno & Sin trastorno & $p$ \\
\hline & $27.75 \pm 7.59$ & $8.7 \pm 4.43$ & $<0.0001$ \\
\hline $\begin{array}{l}\text { BES (16-SF) } \\
\begin{array}{l}\text { Depresión } \\
\text { (Hamilton) }\end{array}\end{array}$ & $16.25 \pm 6.41$ & $8.9 \pm 3.5$ & $<0.0001$ \\
$\begin{array}{l}\text { Ansiedad } \\
\text { (Beck) }\end{array}$ & $27.25 \pm 4.22$ & $11.9 \pm 9.82$ & $<0.001$ \\
\hline
\end{tabular}

monoterapia aún no representa el tratamiento más usual en el manejo de la diabetes tipo 2. También es posible observar esto en nuestro estudio, en el cual la insulina como monoterapia no representa el principal esquema de tratamiento. ${ }^{19,20}$

En lo que respecta a la prevalencia de trastorno depresivo en la población diabética de nuestro estudio, ésta es considerablemente mayor en comparación con la reportada en otros estudios donde se ha descrito una prevalencia no mayor de $55 \%{ }^{21}$ empero sí existen estudios tanto en México como en otras latitudes que concuerdan con nuestros resultados donde la prevalencia del trastorno depresivo supera a más de la mitad de la población estudiada. ${ }^{21-25}$

Diversos estudios y metaanálisis han señalado que la diabetes es un factor de riesgo de desarrollo de depresión en comparación con aquellos sujetos sin la enfermedad, con un OR de casi el doble. Sin embargo, algunos de estos estudios han destacado que la diabetes sólo es un factor de riesgo que en conjunto con otros factores como la obesidad, tabaquismo, alcoholismo, entre otros, son los que desencadenan trastornos depresivos. ${ }^{25,26}$

Sin embargo, esto puede ser derivado no sólo de factores psicosociales, sino que existen alteraciones biológicas ligadas a la diabetes que pueden contribuir directamente al desarrollo de depresión como la reducción en la síntesis de 5-hidroxitriptamina (5-HT) y de la dopamina a expensas de la pérdida de la regulación de la glucemia. Si bien la dopamina puede ser estimulada para incrementar su producción con actividad física, no sucede lo mismo con la síntesis de 5-HT. 27,28 Por otro lado, también se han documentado cambios en la síntesis de cortisol, modificaciones del eje hipotálamo-hipófisis-adrenal en los pacientes diabéticos incluyendo también cambios en la concentración del factor neurotrófico derivado del cerebro (BDNF). ${ }^{29,30}$ Siendo todos estos factores partícipes de la depresión. ${ }^{31,32}$

Por lo anterior, es necesario considerar que parte de la prevención de los trastornos depresivos en los pacientes diabéticos es un adecuado control glucémico; sin embargo, es común una falta de adherencia a la dieta y al tratamiento farmacológico en los pacientes, por lo que hay una constante pérdida de los controles glucémicos. ${ }^{33}$ Estas alteraciones glucémicas pueden a su vez convertirse en un factor clave para disminuir la síntesis de 5-HT y BDNF que, como se mencionó previamente, son condiciones fisiológicas para el desarrollo de la depresión. ${ }^{31,32}$

Cabe señalar que aunque nuestra población estudiada se encuentra cercana a estar conformada por adultos mayores, cuando se realizó un análisis de la media de la edad de la población con o sin depresión, ninguno de los dos grupos clasificaba como adulto mayor. Ahora bien, se ha documentado una correlación entre el incremento de la edad con la incidencia de los trastornos depresivos, lo cual explica la diferencia de la media de edad del grupo con y sin depresión en nuestro estudio. ${ }^{34}$ Empero, cuando evaluamos la edad por la presencia del trastorno de atracones, no observamos diferencia de edad y ambos grupos se encontraban por arriba de los 54 años. Esto es importante, ya que elimina el sesgo que se podría generar en nuestro estudio a consecuencia de la edad y la asociación de ésta con la depresión.

En cuanto a la presencia de ansiedad en la población de pacientes diabéticos estudiados también resultó ser más alta que la reportada en estudios en otros países; sin embargo, es similar a lo descrito en México. ${ }^{24,35}$ Aunque no se tiene aún claro el mecanismo fisiológico por el cual se entrelazan la diabetes y el desarrollo de la ansiedad, en la actualidad la explicación versa en relación con los cambios sociales, emocionales y psicológicos que se desencadenan con la diabetes como factores de riesgo de desarrollo de la ansiedad. ${ }^{36}$ Por lo que la diabetes ha sido asociada como un factor de riesgo considerable de desarrollo de síntomas y cuadros ansiosos en metaanálisis. ${ }^{37,38}$ De ahí que nuestros resultados sobre ansiedad puedan ser explicados como consecuencia del diagnóstico de diabetes, lo cual es importante debido a que la ansiedad se asocia con cambios en los hábitos alimenticios y la presencia de trastornos de la alimentación. 39,40

Lo anterior es relevante, ya que como se puede observar todas las personas que mostraron algún grado de ansiedad fueron positivas para trastorno de atracones y viceversa. Por tal motivo, en nuestro estudio se ratifica la relación entre la ansiedad y los trastornos de la alimentación.

Se detectó un alto porcentaje de personas con trastorno de atracones, el cual es similar a lo reportado en diversos estudios, incluso nuestro resultado se asemeja a los niveles más altos de trastorno de atracones en la población diabética, según lo descrito en un metaanálisis que incluyó 10 estudios sobre desórdenes alimenticios en pacientes diabéticos. ${ }^{41}$ Cabe señalar que aparte de los trastornos de ansiedad, los trastornos también se han asociado con el desarrollo de trastorno de atracones en diversas poblaciones. ${ }^{40,42,43}$ 
La presencia de trastorno de atracones se ha asociado directamente con falla en el control glucémico de los pacientes con diabetes y por ende, con un aumento en la aparición de comorbilidades como la depresión y ansiedad, como ya se ha mencionado. ${ }^{44}$ Por lo anterior, podemos decir que los cambios inducidos en la glucemia por la diabetes tipo 2 propician el desarrollo de condiciones fisiológicas para la aparición de trastornos mentales tales como los depresivos y aunado a los cambios socioemocionales, que permiten la instauración de cuadros de ansiedad, se potencia la aparición de trastornos alimenticios como el atracón.

Si bien este estudio es sólo un análisis de una pequeña muestra, permite identificar diversas situaciones que deben ser abordadas en el estudio y tratamiento de la diabetes. Ya que como se ha descrito, la diabetes y los cambios glucémicos generan un ciclo fisiológico que propicia alteraciones en la alimentación y conductuales que conllevan a nuevos cambios en la alimentación y por ende, a cambios glucémicos perpetuando un ciclo que debe ser atendido e interrumpido con intervención especializada.

\section{CONCLUSIONES}

La diabetes mellitus se caracteriza también por presentar alteraciones de tipo psiquiátrico como los trastornos depresivos, ansiosos y el trastorno de atracones; este último a su vez es perpetuado por el desarrollo de trastornos depresivos y ansiosos que se han descrito como posibles complicaciones biológicas de la diabetes mellitus tipo 2 .

\section{REFERENCIAS}

1. American Diabetes Association. 2. Classification and diagnosis of diabetes: standards of medical care in diabetes-2019. Diabetes Care. 2019; 42 (Suppl 1): S13-S28.

2. Hernández-Romieu AC, Elnecavé-Olaiz A, Huerta-Uribe N, ReynosoNoverón N. Análisis de una encuesta poblacional para determinar los factores asociados al control de la diabetes mellitus en México. Salud Publica Mex. 2011; 53 (1): 34-39.

3. Iglay K, Hannachi H, Joseph Howie P, Xu J, Li X, Engel SS et al. Prevalence and co-prevalence of comorbidities among patients with type 2 diabetes mellitus. Curr Med Res Opin. 2016; 32 (7): 12431252.

4. Katon WJ. The comorbidity of diabetes mellitus and depression. Am J Med. 2008; 121 (11 Suppl 2): S8-S15.

5. Violante ORM. Obesidad y diabetes tipo 2 en el niño. Una nueva epidemia. Rev Endocrinol Nutr. 2001; 9 (2): 103-106.

6. Cabrera-Pivaral CE, González-Pérez G, Vega-López MG, Arias-Merino ED. Impacto de la educación participativa en el índice de masa corporal y glicemia en individuos obesos con diabetes tipo 2. Cad Saúde Pública. 2004; 20 (1): 275-281.

7. Hernández-Escalante V, Trava-García M, Bastarrachea-Sosa R, Laviada-Molina H. Trastornos bioquímicos y metabólicos de la bulimia nervosa y la alimentación compulsiva. Salud Mental. 2003; 26 (3): 9-15.
8. Association AP. Guía de consulta de los criterios diagnósticos del DSM $-5^{\circledR}$ [Spanish Edition of the Desk Reference to the Diagnostic Criteria From DSM- ${ }^{\circledR}$ ] American Psychiatric Pub; 2014.

9. García Palacios A. El trastorno por atracón en el DSM-5. C Med Psicosom. 2014; 110: 70-74.

10. Goode RW, Cowell MM, Mazzeo SE, Cooper-Lewter C, Forte A, Olayia Ol et al. Binge eating and binge-eating disorder in Black women: a systematic review. Int J Eat Disord. 2020; 53 (4): 491-507.

11. Dmitrzak-Węglarz M, Tyszkiewicz-Nwafor M, Duda J, Paszyńska E, Matuszak-Wojciechowska L, Hanć T et al. Abnormal body weight and food-related behavior in school-aged children as measured by the Children's Binge Eating Disorder Scale. Clin Child Psychol Psychiatry. 2020; 25 (2): 304-319.

12. Gormally J, Black S, Daston S, Rardin D. The assessment of binge eating severity among obese persons. Addict Behav. 1982; 7 (1): 47-55.

13. Grupski AE, Hood MM, Hall BJ, Azarbad L, Fitzpatrick SL, Corsica JA. Examining the Binge Eating Scale in screening for binge eating disorder in bariatric surgery candidates. Obes Surg. 2013; 23 (1): 1-6.

14. Ruiz TA, Silva IH, Miranda CE. Diagnóstico clínico y psicométrico de la depresión en pacientes de medicina general. Rev Méd Chile. 2001; 129: 627-633.

15. Vázquez OG, Castillo ER, García AM, Ponce JLA, Avitia MAA, Aguilar SA. Propiedades psicométricas del inventario de ansiedad de Beck (BAI) en pacientes con cáncer. Psicooncología. 2015; 12 (1): 51-58.

16. Sanz J, García-Vera MP, Fortún M. El "Inventario de ansiedad de Beck" (BAl): propiedades psicométricas de la versión española en pacientes con trastornos psicológicos. Psicología Conductual. 2012; 20 (3): 563-583.

17. INEGI. Población. Disponible en: https://www.inegi.org.mx/temas/ estructura/2015.

18. Hernández-Ávila M, Gutiérrez JP, Reynoso-Noverón N. Diabetes mellitus en México: el estado de la epidemia. Salud Pública Méx. 2013; 55: s129-s136.

19. Akirov A, Dicker D, Shochat T, Shimon I. Mortality risk in admitted patients with diabetes mellitus according to treatment. I Diabetes Complications. 2016; 30 (6): 1025-1031.

20. Tandon R, Luxami V, Dosanjh HS, Tandon N, Paul K. Insulin therapy for diabetes epidemic: a patent review. Curr Drug Deliv. 2018; 15 (6): 777-794.

21. Castillo-Quan JI, Barrera-Buenfil DJ, Pérez-Osorio JM, ÁlvarezCervera FJ. Depresión y diabetes: de la epidemiología a la neurobiología. Rev Neurol. 2010; 51 (6): 347-359.

22. Castillo-Quan JI. Insulin resistance, hypercortisolism, polycystic ovarian syndrome, and depression-nonrandom associations? Fertily Steril. 2008; 89 (4): 1029-1030.

23. Pineda N, Bermúdez V, Cano C, Mengual E, Romero J, Medina M et al. Niveles de Depresión y Sintomatología característica en pacientes adultos con diabetes mellitus tipo 2. AVFT. 2004; 23 (1): 74-78.

24. Rivas-Acuña V, García-Barjau H, Cruz-León A, Morales-Ramón F, Enríquez-Martínez RM, Román-Alvarez J. Prevalencia de ansiedad y depresión en las personas con diabetes mellitus tipo 2. Salud en Tabasco. 2011; 17 (1-2): 30-35.

25. Mezuk B, Eaton WW, Albrecht S, Golden SH. Depression and type 2 diabetes over the lifespan: a meta-analysis. Diabetes Care. 2008; 31 (12): 2383-2390.

26. Anderson RJ, Freedland KE, Clouse RE, Lustman PJ. The prevalence of comorbid depression in adults with diabetes: a meta-analysis. Diabetes Care. 2001; 24 (6): 1069-1078.

27. Gomez R, Vargas CR, Wajner M, Barros HM. Lower in vivo brain extracellular GABA concentration in diabetic rats during forced swimming. Brain Res. 2003; 968 (2): 281-284.

28. Rowland NE, Bellush LL. Diabetes mellitus: stress, neurochemistry and behavior. Neurosci Biobehav Rev. 1989; 13 (4): 199-206. 
29. Chiodini I, Adda G, Scillitani A, Coletti F, Morelli V, Di Lembo S et al. Cortisol secretion in patients with type 2 diabetes: relationship with chronic complications. Diabetes Care. 2007; 30 (1): 83-88.

30. Fujinami A, Ohta K, Obayashi H, Fukui M, Hasegawa G, Nakamura N et al. Serum brain-derived neurotrophic factor in patients with type 2 diabetes mellitus: Relationship to glucose metabolism and biomarkers of insulin resistance. Clin Biochem. 2008; 41 (10-11): 812-817.

31. Yu H, Chen ZY. The role of BDNF in depression on the basis of its location in the neural circuitry. Acta Pharmacol Sin. 2011; 32 (1): 3-11.

32. Herbert J. Cortisol and depression: three questions for psychiatry. Psychol Med. 2013; 43 (3): 449-469.

33. Ortiz M, Ortiz E, Gatica A, Gómez D. Factores psicosociales asociados a la adherencia al tratamiento de la diabetes mellitus tipo 2. Terapia Psicológica. 2011; 29 (1): 5-11.

34. Matud MP, Guerrero K, Matías RG. Relevancia de las variables sociodemográficas en las diferencias de género en depresión. Int J Clin Health Psychol. 2006; 6 (1): 7-21.

35. Raffield LM, Brenes GA, Cox AJ, Freedman BI, Hugenschmidt CE, Hsu FC et al. Associations between anxiety and depression symptoms and cognitive testing and neuroimaging in type 2 diabetes. J Diabetes Complications. 2016; 30 (1): 143-149.

36. Bystritsky A, Danial J, Kronemyer D. Interactions between diabetes and anxiety and depression: implications for treatment. Endocrinol Metab Clin North Am. 2014; 43 (1): 269-283.
37. Smith KJ, Beland M, Clyde M, Gariepy G, Page V, Badawi G et al. Association of diabetes with anxiety: a systematic review and metaanalysis. J Psychosom Res. 2013; 74 (2): 89-99.

38. Hasan SS, Clavarino AM, Mamun AA, Kairuz T. Anxiety symptoms and the risk of diabetes mellitus in Australian women: evidence from 21-year follow-up. Public Health. 2016; 130: 21-28.

39. López-Aguilar X, Mancilla-Díaz JM, Vázquez-Arévalo R, Téllez-Girón MTO, Franco-Paredes K, Alvarez-Rayón GL. Factores predictores del atracón alimentario en una muestra comunitaria de mujeres mexicanas. JBHSI. 2010; 2 (1): 25-38.

40. Cuadro E, Baile JI. El trastorno por atracón: análisis y tratamientos Rev Mex Trastor Aliment. 2015; 6: 97-107.

41. Abbott S, Dindol N, Tahrani AA, Piya MK. Binge eating disorder and night eating syndrome in adults with type 2 diabetes: a systematic review. J Eat Disord. 2018; 6: 36.

42. Morales Pernalete AR, Gordillo Gutierrez CA, Pérez Alvarado C], Marcano Flores DA, Pérez Pérez FA, Flores Navas HL et al. Factores de riesgo para los trastornos por atracón (TA) y su asociación con la obesidad en adolescentes. Gac Med Mex. 2014; 150 (Suppl: 1): 125-131.

43. Guisado JA, Vaz FJ. Aspectos clínicos del trastorno por atracón "binge eating disorder". Rev Asoc Esp Neuropsiq. 2001; (77): 27-32.

44. Racicka E, Bryńska A. Eating disorders in children and adolescents with type 1 and type 2 diabetes: prevalence, risk factors, warning signs. Psychiatr Pol. 2015; 49 (5): 1017-24. 